\title{
Low Cost S-Band Phase Modulator for Modern TTC Application
}

\author{
Carlos Hidalgo García ${ }^{\# 1}$, Amparo Herrera Guardado ${ }^{\# 2}$, Javier Cabo Freixedas ${ }^{* 3}$ \\ \#DICOM, UNICAN, Spain \\ *Thales Alenia Space Spain, Spain \\ $\left\{{ }^{1}\right.$ hidalgoc, ${ }^{2}$ amparo.herrera $\} @$ unican.es, ${ }^{3}$ javier.cabofreixedas@thalesaleniaspace.com
}

\begin{abstract}
This paper describes the design, simulation and practical results of a phase modulator based in an hybrid coupler and two varactor diodes in the band from $2.0 \mathrm{GHz}$ to $3.0 \mathrm{GHz}$ for TTC proposes. A minimum modulation index of 1.22 was obtained at the highest frequency to 1.57 at the lowest. This modulator consist of a two stages hybrid coupler with varactor diodes. Simulation is performed using ADS 2016.01. Even both varactor diodes are feed by the same modulation signal, each of them has his own voltage input for correct varactor impairments. In the measures, excellent isolation is achieved for the $1^{\text {st }}$ and $4^{\text {th }}$ port of the ring.
\end{abstract}

Keywords - Phase modulation, varactors, hybrid integrated circuits, couplers.

\section{INTRODUCTION}

In TTC communications, a modulated microwave signal is required, and most of high frequency applications main concerns are component's size and weight. A new type of transponders were designed, using traditional phase modulation or via a data relay satellite using spread-spectrum in PSK modulation with suppressed carrier. Difficulties mainly related to the propagation channel and the required high QoS (Quality of Service).

Phase modulated signals allow on-board coherent demodulation of the received signal. PM signals show high resistance to channel non-linarity thanks to their constant envelope and, moreover, the multiplicity of modulation schemes offer more flexibility to cope with propagation conditions [1].

Phase modulators is the solution for these types of high profile applications because it effectively reduces the weight and size of the transmitter. As to the details in this case, varactor diodes have been utilized in microwave phase shifters by varying the junction capacitance of a diode as a function of an applied voltage across it. Joining it with an hybrid coupler in these phase modulator, a relatively large phase shift, as a function of applied voltage, is obtained.

The requirements of a microwave phase modulator are ideally not only to maintain the linear relationship between the phase and the modulation signal voltage, but also to have no change in the carrier amplitude with a wide-band modulating video and large modulation index. It is difficult to satisfy both amplitude and phase requirements in a modulator where a four terminal network is involved [2][3].
As part of a background concept, if the carrier signal is phase modulated, the resultant signal is [4]:

$$
\begin{aligned}
& \quad e(t)=J_{o}(m) \cos \left(\omega_{c} t\right)-J_{1}(m)\left(\cos \left(\left(\omega_{c}-\omega_{m}\right) t\right)-\right. \\
& \left.\cos \left(\left(\omega_{c}+\omega_{m}\right) t\right)\right)+J_{2}(m)\left(\cos \left(\left(\omega_{c}-2 \omega_{m}\right) t\right)+\right. \\
& \left.\cos \left(\left(\omega_{c}+2 \omega_{m}\right) t\right)\right) \ldots
\end{aligned}
$$

Where:

$J_{n}(m)$ is the Bessel function of the first kind, $\mathrm{n}$ order

$\omega_{c}$ is the carrier frequency

$\omega_{m}$ is the modulating signal frequency

$m$ is the modulation index

In phase modulation, the modulation index is equal to the peak phase swing in radians independent of its frequency. For a small index, the level of the first sideband is proportional to the peak phase swing of the carrier produced by the modulating signal. Therefore, for a linear phase modulator using small modulation index, the modulating signal is effectively frequency shifted to the first sideband of the carrier signal [4]. For cost reasons, it is desirable to have an index as large as possible, while maintaining linearity and bandwidth. To maximize the carrier to sideband conversion efficiency, careful consideration of the choice of varactor diode and the design of the RF circuit is necessary.

In this paper, a low cost and low size phase modulator using hybrid coupler and varactor diodes as part of the final hybrid circuit is proposed. Here we use the two-staged hybrid coupler to isolate the input and output signals, and two varactor diodes to reflect the RF signal, resulting in a variable phase shift range of $90^{\circ}(m=1.57)$ at $2.3 \mathrm{GHz}$. By using hybrid technology and Rogers 4003 as substrate $(\varepsilon=3.55, H=12$ mils, $\tan \delta=$ 0.0021 ), the designed phase modulator has a size limit of 50 $\mathrm{mm} \times 25 \mathrm{~mm}$. This substrate is selected because of its low $\varepsilon$ variation with temperature $\left(-30^{\circ}\right.$ to $\left.85^{\circ}\right)$.

\section{Design Methodology}

To design the phase modulator, reflexive phase shifter technique is used. Maynly consist of a $3 \mathrm{~dB}$ hybrid coupler and varactor diodes, as mentioned above.

\section{A. Two-stage Hybrid Coupler}

The coupler can consist of a single or a multiple stages. A single stage coupler has a relatively narrow bandwidth, 
although, a higher bandwidth is achieved using a two staged hybrid coupler, in terms of return loss thereby increasing the VSWR at the operating frequency band.

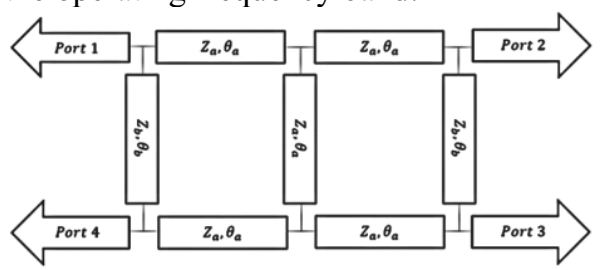

Fig. 1. Two-staged Hybrid Coupler

Where:

$Z_{0}$ is the reference impedance, $50 \Omega$

$Z_{a}=\frac{Z_{0}}{\sqrt{2}} \Omega$

$Z_{b}=\frac{Z_{0}}{\sqrt{2}-1} \Omega$

$\theta_{a}=\theta_{b}=\frac{\lambda}{4}$

Fig. 1 shows different values of impedances at the various brands of the coupler. In accordance to the impedances, the length and width of the brands changes. Lengths and widths were calculated using line calculator method and substrate characteristics. The Scattering matrix of a one-stage branch-line coupler are given by:

$$
[S]=\left[\begin{array}{llll}
S_{11} & S_{12} & S_{13} & S_{14} \\
S_{21} & S_{22} & S_{23} & S_{24} \\
S_{31} & S_{32} & S_{33} & S_{34} \\
S_{41} & S_{42} & S_{43} & S_{44}
\end{array}\right]=\frac{1}{\sqrt{2}}\left[\begin{array}{cccc}
0 & 1 & j & 0 \\
1 & 0 & 0 & j \\
j & 0 & 0 & 1 \\
0 & j & 1 & 0
\end{array}\right]
$$

There is an equal power split of the incident signal at port 1 to ports 2 and 3 . No signal propagates directly from port 1 to port $4\left(S_{14}=S_{41}=0,(2)\right)$. The signal reaching port 2 has a phase delay $\Delta \phi$, while for the port one reaching port 3 the $\Delta \phi+$ $90^{\circ}\left(S_{21}=S_{12}=\frac{1}{\sqrt{2}} ; S_{13}=S_{31}=\frac{j}{\sqrt{2}}=S_{21} e^{\frac{j \pi}{2}}\right)$. The signals at the ports 2 and 3 are reflected back to port 1 and 4 by the two identical reflective terminations after a delay $\alpha$. The reflected wave from port 2 reaches port 1 with the total delay of $2 \Delta \phi+$ $\alpha$, whereas the signal reflected from port 3 , gets at port 1 with a delay of $2 \Delta \phi+180^{\circ}+\alpha$. The signals are in opposite phase and therefore cancel. However, the signal at port 4 is the superposition of signals reflected from the ports 2 and 3 both having the phase $2 \Delta \phi+90^{\circ}+\alpha$ interfere constructively [5].

\section{B. Impedance Variation of the Reflective Load Based on Varactor Diodes}

The desired phase modulation is achieved by varying the phase of the reflection coefficient $(\Gamma)$ of the reflective load $\left(Z_{T}\right)$ obtained by varying the impedance of the reflective load including a varactor. The resulting phase variation $(\Delta \phi)$ is given by [6]:

$$
\Delta \phi=2\left|\arctan \left(\frac{Z_{\max }}{Z_{o}}\right)-\arctan \left(\frac{Z_{\min }}{Z_{o}}\right)\right|
$$

Where $Z_{\max }$ and $Z_{\min }$ are the maximum and minimum reflective load and $Z_{o}$ the input/output source impedance. By applying a modulated signal to the reverse bias of the varactor, the phase change is observed due to the explanation above. The commercial selected varactor diode is DH76022, with a reverse breakdown voltage from $0 \mathrm{~V}$ to $20 \mathrm{~V}$, decreasing its capacitance from $5.2 \mathrm{pF}$ to $0.9 \mathrm{pF}$.

Adaptation network is needed in ports 2 and 3 to centre the reflection coefficient in the desired values. To correct possible variations of the varactor capacitances, an additional source for each varactor is added.

\section{Proposed Phase Modulator}

\section{A. Design Consideration}

The phase modulator proposed should have a balance between cost, size and parameters. The main objectives are:

Band-width: $2.0 \mathrm{GHz}-3.0 \mathrm{GHz}$ (S-Band)

Modulation index: 1.22

Index stability: $\pm 3 \%\left(-30^{\circ} \mathrm{C}\right.$ to $\left.85^{\circ} \mathrm{C}\right)$

Insertion Loss: $2 \mathrm{~dB}$

Modulation Band-width: $10 \mathrm{kHz}$ to $1 \mathrm{MHz}$

Area: $19 \mathrm{~mm}$ x $50 \mathrm{~mm}$

The modulated signal is obtained at the $4^{\text {th }}$ port and the two varactors are added at the $2^{\text {nd }}$ and $3^{\text {rd }}$ port of the hybrid coupler. Maximum allowable bias voltage is $5 \mathrm{~V}$ which should provide minimum phase change of 70 degrees.

\section{B. Implementation}

First of all, the hybrid coupler and the adaptation network is designed and simulated in ADS2016.01 (Fig. 2) using the theory above and the substrate characteristics. The comparison between simulation and measures are shown in Fig. 3.

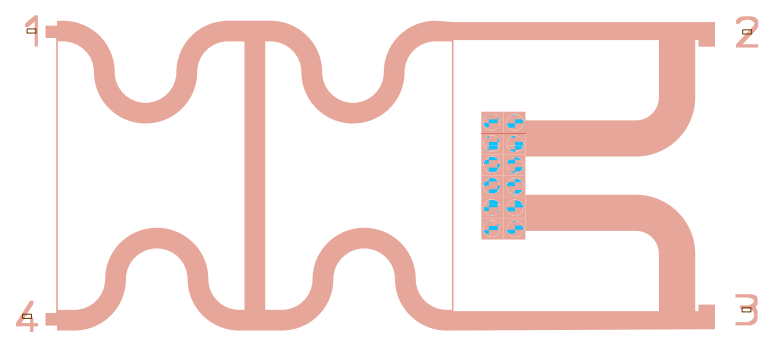

Fig. 2. Hybrid Coupler plus Adaptation Network in Ports 2 and 3. Curved lines are needed for size requirements

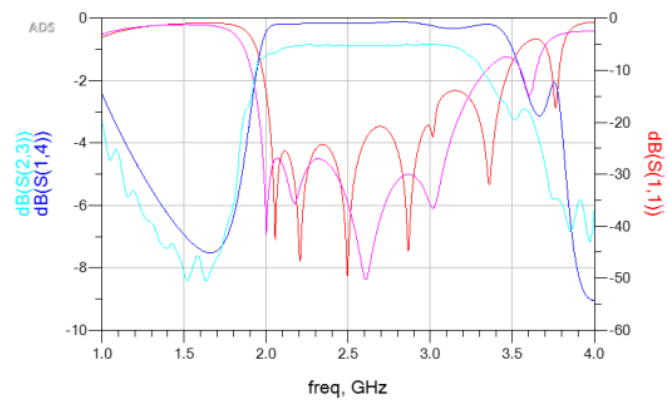

Fig. 3. Scattering parameters. Top graph: $\mathrm{S}(1,1)$ simulated in red, measured in pink; $S(2,1)$ simulated in blue, measured in cyan 


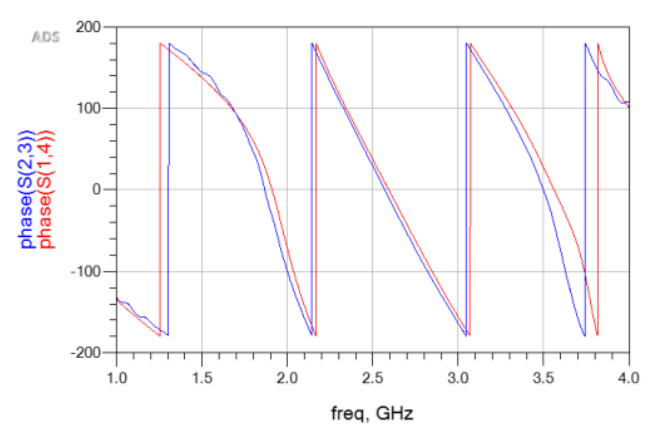

Fig. 4. Scattering parameters. Phase $(\mathrm{S}(2,1))$ simulated in red, measured in blue

A diminution on the band width is observed. The simulation results do not include losses on the microstrip conductors or the connectors. Next step was include the varactor diodes and bias network at ports 2 and 3 and capacitors at ports 1 and 4 for adaptation purposes. A photo of the PCB with the final layout is shown in Fig. 6. The varactor diode was selected among the available for its capacitance values with the bias change, its model is not accurate demonstrated in the I-V graph Fig. 5.
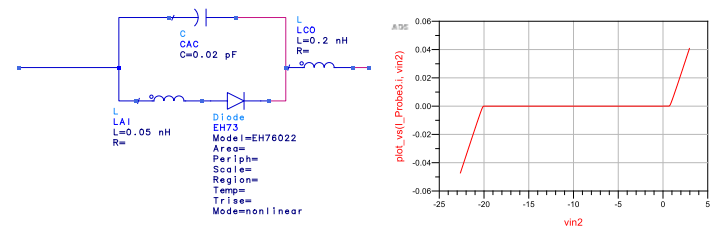

Fig. 5. Varactor Diode's Schematic Model and I-V Graph

PCB is made in Rogers 4003. The process consist of four steps: substrate holing, metallizing, laser-engraved pattern and all the required components are soldered on the board. All the resistors and capacitors are SMD type. All pads included in Fig. 6 layout.

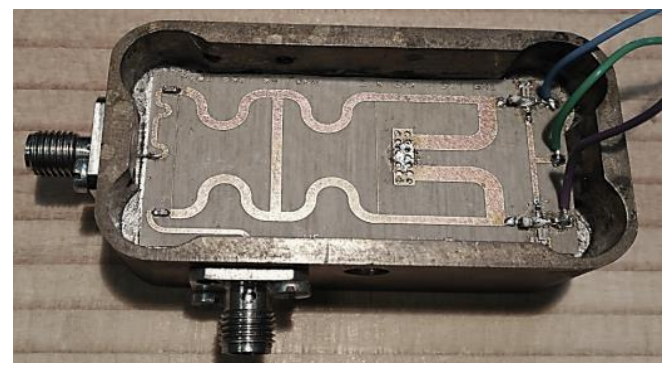

Fig. 6. Phase Modulator's PCB

\section{SimUlation AND EXPERIMENTAL RESUlts}

To observe the phase shift with voltage in the proposed band, discrete voltage values from $0 \mathrm{~V}$ to $10 \mathrm{~V}$ are applied at the modulated signal's port, while $\mathrm{S}$ parameters are measured between the ports 1 and 4 of the phase modulator. $V_{d_{1}}$ and $V_{d_{2}}$ could be used to correct varactor impairments.

In Fig. 7, the comparison between the simulation and measurements $\mathrm{S}(2,1)$ and $\mathrm{S}(1,1)$ are shown :

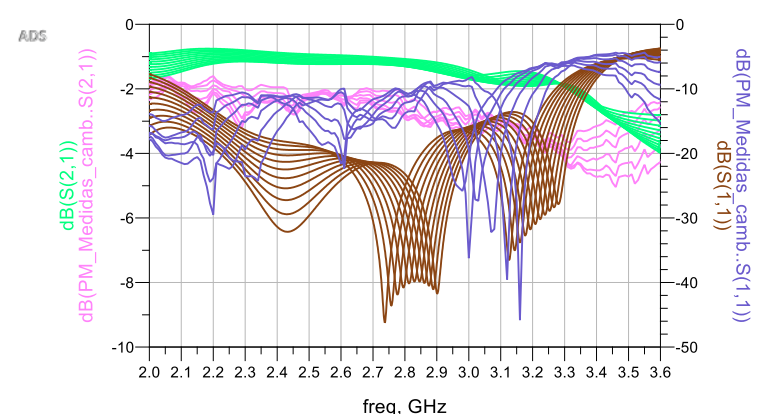

Fig. 7. Comparison between the Simulation and Measurements of the S Parameters of the Phase Modulator

Adaptation is under $-8 \mathrm{~dB}$ in the bandwidth of interest and losses are lower than $-3.2 \mathrm{~dB}$. Regarding to the phase behaviour, two main studies had been done: first, the phase deviation with the discrete values of $V_{m}$ to show the bias point necessary to obtain the desirable modulation index; then the phase nonlinearity with the frequency, to show the achievable bandwidth.

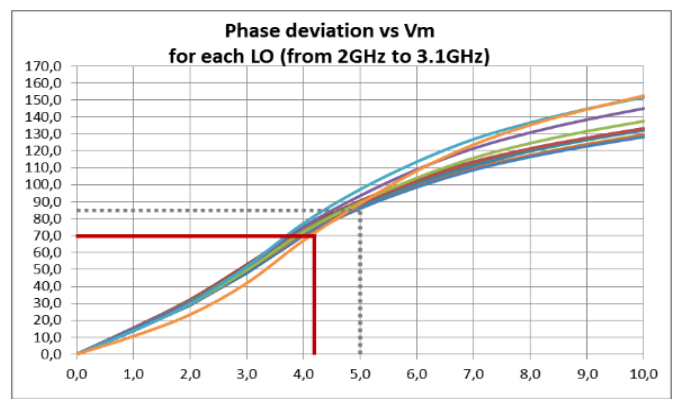

Fig. 8. Measures of the Phase Deviation (in Degrees; the Modulation Index is this Value in Radians) vs. Vm for each LO (from $2 \mathrm{GHz}$ to $3.1 \mathrm{GHz}$ )

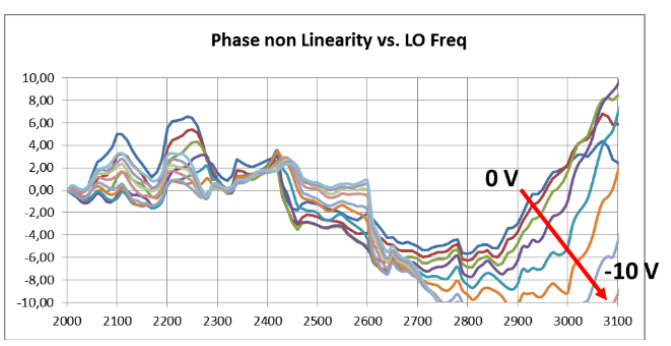

Fig. 9. Measures of the Phase Non Linearity (Degrees) vs. LO Freq for each Discrete Vm Value

As shown in the Fig. 8, the phase modulator can reach a modulation index of $1.22\left(70^{\circ}\right)$ with $4.15 \mathrm{~V}$, that is suitable due to the voltage limitations, reaching an index of $1.48\left(85^{\circ}\right)$ with $5 \mathrm{~V}$. In the other hand, as seen in Fig. 9 a phase non linearity of $10 \%$ is reached for frequencies over $2.6 \mathrm{GHz}$, being this value the top limit of the bandwidth.

Phase modulator is then tested using both oscilloscope and spectrum analyser. In the oscilloscope the states of the modulated signal are shown, in the spectrum analyser, the carrier-first side band difference is measured. In the first case, Fig. 10, carrier gets $0 \mathrm{dBm}$ and is fixed in $2.3 \mathrm{GHz}$, modulation signal gets four states corresponding with $0 / 1 / 2 / 3 \mathrm{~V}_{\mathrm{pp}}$ and an offset of $-1.5 \mathrm{~V}$ (maximum values of the arbitrary signal 
generator, Agilent $81180 \mathrm{~B}), 100 \mathrm{kHz}$. The modulated signal is shown:

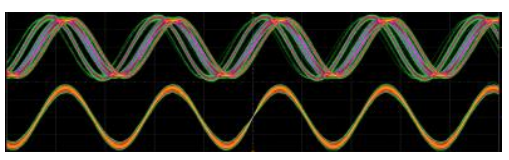

Fig. 10. Top Image: 4 States in the Modulated Signal. Down Image: Carrier

In the second case, Fig. 11, carrier gets $0 \mathrm{dBm}$ and is swept from $2 \mathrm{GHz}$ to $2.6 \mathrm{GHz}$ with $0.3 \mathrm{GHz}$ steps. Modulation signal keeps parameters explained above. In Table. 1, the values of carrier - first side band relation:

Table 1 Carrier - First Side Band Relation (4 States)

\begin{tabular}{|l|l|l|l|}
\hline States & $\mathbf{2 ~ G H z}$ & $\mathbf{2 . 3} \mathbf{~ G H z}$ & $\mathbf{2 . 6} \mathbf{~ G H z}$ \\
\hline $0 / 1 / 2 / 3 \mathrm{~V}$ & $-13.2 \mathrm{dBc}$ & $-12.6 \mathrm{dBc}$ & $-12.9 \mathrm{dBc}$ \\
\hline $0 / 0.8 / 1.6 / 2.5 \mathrm{~V}$ & $-15.2 \mathrm{dBc}$ & $-13.7 \mathrm{dBc}$ & $-14 \mathrm{dBc}$ \\
\hline $0 / 0.7 / 1.4 / 2 \mathrm{~V}$ & $-15.7 \mathrm{dBc}$ & $-14.6 \mathrm{dBc}$ & $-15 \mathrm{dBc}$ \\
\hline $0 / 0.5 / 1 / 1.5 \mathrm{~V}$ & $-17.1 \mathrm{dBc}$ & $-16.5 \mathrm{dBc}$ & $-16.8 \mathrm{dBc}$ \\
\hline
\end{tabular}

When modulation signal is a sine with $3 \mathrm{~V}_{\mathrm{pp}}$ and a variable offset to make the maximum value equal to $0 \mathrm{~V}$. In the next tables, the values of the carrier - first side band relation and corresponding modulation index:

Table 2. Carrier - First Side Band Relation (Sine-Wave Modulation Signal)

\begin{tabular}{|l|l|l|l|}
\hline States & $\mathbf{2 ~ G H z}$ & $\mathbf{2 . 3} \mathbf{~ G H z}$ & $\mathbf{2 . 6} \mathbf{~ G H z}$ \\
\hline $3 \mathrm{~V} \mid \mathrm{V}_{\text {offset }}-1.5$ & $-5.8 \mathrm{dBc}$ & $-5.2 \mathrm{dBc}$ & $-5.2 \mathrm{dBc}$ \\
\hline $2.5 \mathrm{~V} \mid \mathrm{V}_{\text {offset }}-1.25$ & $-7.6 \mathrm{dBc}$ & $-6.8 \mathrm{dBc}$ & $-6.8 \mathrm{dBc}$ \\
\hline $2 \mathrm{~V} \mid \mathrm{V}_{\text {offset }}-1$ & $-10.1 \mathrm{dBc}$ & $-9.1 \mathrm{dBc}$ & $-9.2 \mathrm{dBc}$ \\
\hline $1.5 \mathrm{~V} \mid \mathrm{V}_{\text {offset }}-0.75$ & $-12.2 \mathrm{dBc}$ & $-11.2 \mathrm{dBc}$ & $-11.8 \mathrm{dBc}$ \\
\hline
\end{tabular}

Table 3. Carrier - Modulation Index (Consider Equipment Limitations)

\begin{tabular}{|l|l|l|l|}
\hline States & $\mathbf{2 ~ G H z}$ & $\mathbf{2 . 3} \mathbf{~ G H z}$ & $\mathbf{2 . 6} \mathbf{~ G H z}$ \\
\hline $3 \mathrm{~V} \mid \mathrm{V}_{\text {offset }}-1.5$ & $0.83 \mathrm{rad}$ & $0.85 \mathrm{rad}$ & $0.87 \mathrm{rad}$ \\
\hline $2.5 \mathrm{~V} \mid \mathrm{V}_{\text {offset }}-1.25$ & $0.67 \mathrm{rad}$ & $0.68 \mathrm{rad}$ & $0.70 \mathrm{rad}$ \\
\hline $2 \mathrm{~V} \mid \mathrm{V}_{\text {offset }}-1$ & $0.49 \mathrm{rad}$ & $0.53 \mathrm{rad}$ & $0.54 \mathrm{rad}$ \\
\hline $1.5 \mathrm{~V} \mid \mathrm{V}_{\text {offset }}-0.75$ & $0.31 \mathrm{rad}$ & $0.33 \mathrm{rad}$ & $0.35 \mathrm{rad}$ \\
\hline
\end{tabular}

In Fig. 11, a modulation signal of $100 \mathrm{kHz}$ and 4 cases of amplitude between $0 \mathrm{~V}$ and $3 \mathrm{~V}$ are shown. As long as the number of states decreases or $\mathrm{V}_{\mathrm{pp}}$ of the signal increases, the carrier - first side band relation increases too.

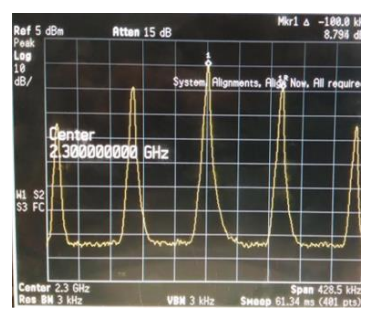

Fig. 11. Example of Carrier (Sine, $2.3 \mathrm{GHz}$ ) - Side Band Relation for each State
Table 4. Performance Comparison (H: Hybrid; the Other Works are Shifters)

\begin{tabular}{|l|l|l|l|l|}
\hline Prop. & Work & {$[$ 7.1] } & {$[$ 7.2] } & {$[$ [8] } \\
\hline Technology & H & H & H & H \\
\hline Die size $\left(\mathrm{mm}^{2}\right)$ & 950 & - & - & 200 \\
\hline Bandwidth/ $\mathrm{F}_{\mathrm{o}}$ & 0.26 & - & - & 0.29 \\
\hline Min. Index & 1.22 & 3.4 & 3.14 & 3.1 \\
\hline Max. Insertion Loss $(\mathrm{dB})$ & 3.2 & 1.5 & 0.6 & 7 \\
\hline Phase Non-Linearity & $10 \%$ & - & - & $2 \%$ \\
\hline Bias (V) & 4.15 & 120 & 10 & - \\
\hline
\end{tabular}

\section{PROBLEMS AND CONCLUSION}

In this paper, a hybrid phase modulator has been presented. The phase modulator uses a hybrid coupler and two varactors in a $19 \mathrm{~mm} \times 50 \mathrm{~mm}$ board. The phase modulator described here is suited for space applications (TTC) bundled with a multiplier, reaching from $2.0 \mathrm{GHz}-2.6 \mathrm{GHz}$, to $6 \mathrm{GHz}-7.8 \mathrm{GHz}$. It got less than $3.5 \mathrm{~dB}$ of insertion loss and good adaptation; a minimum modulation index of 1.22 with less than $10 \%$ of phase error in the worst case, probably, due to the varactor diode's model (under study).

Speaking of latest developments, this phase modulator works in the first half of the $\mathrm{S}$ band, has a fast assembly and a low cost component list. Achieve target modulation index and low insertion losses in the bandwidth, but the phase nonlinearity has to be improved in the second half of the band. The side band rejection is appropriate for our purposes.

\section{ACKNOWLEDGMENT}

This work has been realized thanks to the service provide in the research project "Support and Consulting in the TTC\&RF Active Area" from Thales Alenia Space in Spain. This work was also supported by the Spanish Ministry of Economy and Competitiveness and the European Regional Development Fund (ERDF/FEDER) under research projects TEC201460283-C3-1-R and TEC2017-88242-C3-1-R.

\section{REFERENCES}

[1] R Chaggara, ML Boucheret, C Bazile, E Bouisson, A Ducasse, and JD Gayrard, "Continuous phase modulation for future satellite communication systems in ka band," in Information and Communication Technologies: From Theory to Applications, 2004. International Conference on Proceedings. 2004. IEEE, 2004, pp. 269-270.

[2] D. H. Kuhn, "Study of periodically loaded transmission lines as variable phase shifters and switches," General Electric Co., Syracuse, N. Y., Internal Rept. R60ELS-29. February 24, 1960

[3] H. H. Dawirs and W. G. Swarner, "A very fast, voltage controlled microwave phase shifter,”. Microwave J., vol. V, pp. 99-106, June 1962.

[4] C. J. Mann and M. T. Moore, "Linear varactor upconverters for use in digital microwave radio," IEE Colloquium on Microwave Components in Telecommunications, London, 1988, pp. 10/1-10/4.

[5] A. Gradinaru, "Millimeter-wave scanning-beam phased array antenna". Doctoral and Master theses, University of Toronto, 1988.

[6] H. Zarei, C. T. Charles and D. J. Allstot, "Reflective-Type Phase Shifters for Multiple-Antenna Transceivers," in IEEE Transactions on Circuits and Systems I: Regular Papers, vol. 54, no. 8, pp. 1647-1656, Aug. 2007.

[7] H. Schwarz, "Linear Electronic Phase-Shifter Design," SLAC-PEPNOTE-283, November 1979.

[8] E. Villa, B. Aja, J. Cagigas, E. Artal and L. de la Fuente, "Four-State Full Q-Band Phase Shifter Using Smooth-Ridged Waveguides," in IEEE Microwave and Wireless Components Letters, vol. 27, no. 11, pp. 995-997, Nov. 2017. 